Nouvelles perspectives en sciences sociales

Revue internationale de systémique complexe et d'études relationnelles

\title{
Modèles formels pour la reproduction des simulations à base d'agents
}

\section{Christophe Sibertin-Blanc}

Volume 5, numéro 2, mai 2010

Sur le thème de la simulation

URI : https://id.erudit.org/iderudit/044080ar

DOI : https://doi.org/10.7202/044080ar

Aller au sommaire du numéro

Éditeur(s)

Prise de parole

ISSN

1712-8307 (imprimé)

1918-7475 (numérique)

Découvrir la revue

Citer cet article

Sibertin-Blanc, C. (2010). Modèles formels pour la reproduction des simulations à base d'agents. Nouvelles perspectives en sciences sociales, 5(2), 135-149.

https://doi.org/10.7202/044080ar
Résumé de l'article

Du fait de la dimension expérimentale des résultats obtenus par simulation informatique, la validation de ces résultats nécessite qu'ils puissent être reproduits. Cela suppose que le dispositif concret à partir desquels ils sont obtenus, à savoir le logiciel dont les exécutions fournissent les sorties qui sont observées, puisse donner lieu à de nouvelles implantations. Bien qu'elle soit indispensable à maints égards, il s'avère que cette "réplication" des modèles de simulation est le plus souvent problématique; cet article propose de présenter ces modèles sous la forme de systèmes afin d'en faciliter de nouvelles implantations. 


\title{
Modèles formels pour la reproduction des simulations à base d'agents
}

\author{
Christophe Sibertin-Blanc ${ }^{\mathrm{I}}$ \\ Université de Toulouse 1 \\ Institut de recherche en informatique de Toulouse
}

\section{Introduction}

7 ans le domaine des sciences sociales, la simulation à base d'agents est une pratique qui se développe assez largement et est reconnue comme a third way for doing science ${ }^{2}$. Elle comporte un aspect déductif - le modèle qui donne lieu à des simulations est un objet formel sur lequel des inférences et des calculs logiquement contrôlés peuvent être réalisés. Elle comporte aussi un aspect expérimental puisque les simulations sont destinées à acquérir des résultats nouveaux sur le comportement de ce qui est modélisé; ces résultats ne peuvent pas être obtenus de façon analytique, du fait des phénomènes d'émergence qui résultent de

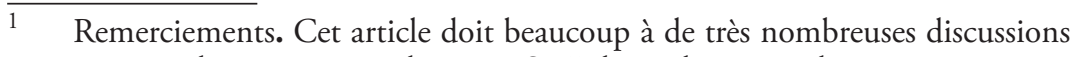
avec tous les participants du projet SocLab, et plus particulièrement Françoise Adreit, Pascal Roggero et Claude Vautier, ainsi qu’à des échanges très enrichissants dans le cadre du projet ANR COSMAGENS, notamment avec Denis Phan.

2 Robert Axelrod, "Advancing the Art of Simulation in the Social Sciences ", dans Rosaria Conte, Rainer Hegselmann et Pietro Terna (dir.), Simulating Social Phenomena, Berlin, Springer-Verlag, 1997, p. 21-40. 
la multiplicité des (types d') interactions entre les éléments qui sont en jeu ${ }^{3}$. Se pose donc le problème de la reproduction des résultats de ces simulations, qui ne peuvent être considérés comme valides que s'ils sont reproduits: "unreplicated simulation models and their results can not be trusted - as with other kinds of experiment, simulations need to be independently replicated. [...] aligning models is very difficult, but very revealing" 4 .

Mais de quelle nature est cette réplication ou reproduction? Tout processus visant à obtenir des résultats scientifiques au moyen de simulations met en jeu quatre éléments. Il y a d'abord, dans un certain domaine, l'objet qui a été repéré comme digne d'intérêt et qui constitue la cible du modèle. Le modèle conceptuel de cette cible en est la représentation sur laquelle se fonde son étude, représentation plus ou moins bien fondée sur une théorie des sciences sociales et qui est, en tout état de cause, exprimée en langage naturel. Le modèle formel est une traduction du modèle conceptuel qui a pour fonction d'en évacuer les ambiguïtés (en faisant certains choix réducteurs) et d'être une description, ou spécification, complète et consistante du dispositif dont l'exécution sur ordinateur produira des résultats de simulation. Vient enfin le modèle implanté qui sera le support opérationnel des simulations réalisées sur un ordinateur. Le passage du modèle conceptuel vers le modèle formel et de ce dernier vers le modèle implanté résulte d'une forme de traduction, chacun d'eux étant formulé dans un langage qui lui est propre, avec les procédés de construction de sa syntaxe, le pouvoir

3 Jean-Louis Dessalles, Jean-Pierre Müller et Denis Phan, "Emergence in Multi-Agent Systems: Conceptual and Methodological Issues ", dans Denis Phan et Frédéric Amblard (dir.), Agent-Based Modelling and Simulation in the Social and Human Sciences, Oxford, The Bardwell Press, 2007, p. 327-356.

4 Bruce Edmonds et David Hales, "Replication, Replication and Replication: Some Hard Lessons from Model Alignment, Journal of Artificial Societies and Social Simulation, vol. 6, n 4, 2003, En ligne : http://www.jasss.soc.surrey. ac.uk/6/4/11.html (le 17 avril 2010).

5 Alexi Drogoul, Diane Vanbergue et Thomas Meurisse, " Multi-Agent Based Simulation: Where Are the Agents? ", dans Jaime Sichman, François Bousquet et Paul Davidsson (dir.), Proceedings of MABS 2002 Multi-Agent-Based Simulation, Lecture Notes in Computer Science 2581, Berlin, SpringerVerlag, 2003, p. 1-15. 
d'expression de sa sémantique et ses tests de cohérence interne. En sens inverse, c'est un processus d'induction / de généralisation qui permet de formuler les résultats relatifs au modèle conceptuel à partir de ceux relatifs au modèle formel, et ces derniers à partir d'observations des sorties de simulations du modèle implanté. C'est donc le modèle implanté qui détermine les conditions pratiques de l'expérience qui a permis d'obtenir des résultats, et renouveler l'expérience nécessite de répliquer le modèle implanté, de réaliser une nouvelle implantation du modèle formel.

De nombreux auteurs ont signalé combien cette réplication est difficile. D’une part, les techniques de vérification du génie logiciel qui permettent de s'assurer qu'une implantation est correcte, c'est-à-dire qu'elle respecte ses spécifications, ne sont pas applicables ici : l'essence d'une simulation est de produire des résultats nouveaux, et on ne dispose donc pas de l'oracle qui permettrait, en fonction des entrées, de comparer les sorties obtenues à celles qui sont attendues et devraient être produites . D'autre part, si les articles publiés fournissent généralement une bonne présentation du modèle conceptuel, ce qui est indispensable pour que le lecteur se fasse une idée assez claire de ce dont il s'agit, le modèle formel, qui hérite nécessairement d'une partie de la complexité de la cible du modèle, est plus difficile à présenter si bien que l'on ne dispose pas de suffisamment d'informations pour en dériver une nouvelle implantation qui produise les mêmes résultats que ceux annoncés.

On pourra se référer par exemple à Wilensky ${ }^{7}$ pour une synthèse des bénéfices de la réplication d'un modèle, sur les difficultés rencontrées et sur les questions de validation, à savoir les critères permettant de dire qu'une réplication d'un modèle formel

$6 \quad$ Frédéric Amblard, Juliette Rouchier et Pierre Bommel, "Évaluation et validation de modèles multi-agents ", dans Frédéric Amblard et Denis Phan (dir.), Modélisation et simulation multi-agents, applications pour les sciences de l'Homme et de la société, Londres, Hermes-Sciences \& Lavoisier, 2006, p. 103-120.

7 Uri Wilensky et William Rand, "Making Models Match: Replicating an Agent-Based Model ", Journal of Artificial Societies and Social Simulation, vol. $10, \mathrm{n}^{\mathrm{o}}$ 4, 2007, En ligne : http://www.jasss.soc.surrey.ac.uk/10/4/2.html (le 17 avril 2010). 
confirme ou non les résultats antérieurs associés à ce modèle. Cet article n'abordera pas davantage la question centrale de la " validité » des résultats et de leur statut épistémique, qui met en cause les relations entre la cible du modèle et le modèle conceptuel et celles entre le modèle conceptuel et le modèle formel (qui en est toujours une simplification), ni la légitimité du processus de production des résultats portant sur le modèle formel à partir des sorties du modèle implanté. Nous nous contenterons de proposer une façon de structurer la présentation de modèles formels, en d'autres termes un formalisme (qui est composite, du fait de la diversité des dimensions à prendre en compte), qui vise à favoriser la réplication de leur implantation. C'est la complétude de la description d'un modèle formel qui pose problème, et non pas sa consistance qui, de par la nature du modèle, peut être soumise à des tests qui vérifient l'absence de contradictions.

Introduire une façon de structurer un objet nécessite de passer à un niveau d'abstraction supérieur pour catégoriser les éléments entrant dans sa composition. Ici, nous nous baserons sur des concepts très généraux de la théorie des systèmes. Ce choix est justifié dans la mesure où la cible du modèle peut être analysée comme un système, le propre d'un modèle de simulation à base d'agents étant précisément de se calquer sur la structure de cette cible.

Si un modèle formel est donc la description d'un système, il devra comporter une description d'une part, de sa structure, de ses éléments constitutifs et des relations qu'ils entretiennent, et, d'autre part, de son comportement, ou fonctionnement, à savoir comment il peut ou doit évoluer.

\section{Structure d'un modèle formel et son espace d'états}

Pour modéliser la structure d'un système, il faut donc identifier, à partir du modèle conceptuel, les éléments des trois catégories d'éléments constitutifs de tout système, à savoir : les entités passives, c'est-à-dire les choses de toute nature qui sont manipulées au cours du fonctionnement du système, auxquelles certains traitements sont appliqués, et que l'on appellera les ressources du 
système; les entités actives, ou acteurs, processeurs, qui disposent d'une source d'énergie et sont capables de réaliser un certain travail de façon plus ou moins autonome ${ }^{8}$; et, enfin, les transitions, unités de travail réalisées par des acteurs, mettant en jeu des ressources et dont l'occurrence fait évoluer l'état ou la structure du système. De façon standard, dans un système à base d'agents, les entités passives et actives seront des agents décrits par la donnée de leur nom, des attributs qui leur sont associés et des services (ou fonctions, méthodes) qu'ils sont capables de réaliser (dans le cas des acteurs) ou qui leur sont applicables (dans le cas des ressources); des transitions, quant à elles, seront décrites par leur nom et la description du travail correspondant, à savoir les services des acteurs et des ressources concernés qu'il s'agit d'effectuer. La description de la structure d'un modèle nécessite aussi de décrire les relations que les éléments de ces trois catégories entretiennent les uns avec les autres.

Ces entités peuvent être classées selon leur type, et les types des entités (de la structure) du modèle et les types de leurs relations constituent un méta-modèle qui peut être représenté sous la forme d'un diagramme de classe UML tel que celui de la figure 1 pour le cas, paradigmatique dans le domaine de la simulation sociale, du modèle de ségrégation résidentielle de Schelling ${ }^{9}$. Ce diagramme de classes doit être accompagné de contraintes sémantiques sur la façon dont il peut être instancié; UML permet d'exprimer certaines de ces contraintes par les cardinalités associés aux rôles (par exemple, un individu occupe un et un seul emplacement), mais il y en a bien d'autres : un individu occupe un emplacement qui lui est accessible, la satisfaction d'un individu est la valeur retournée par sa fonction calculSatisfaction,

$8 \quad$ Les êtres animés ont vocation à être des acteurs et les objets à être des ressources, mais pas nécessairement. Dans un modèle de ruissellement, par exemple, les gouttes d'eau qui consomment de l'énergie potentielle pour se déplacer seront modélisées comme des acteurs, à moins qu'elles ne soient agies par un processeur " gravitation " qui exerce l'ensemble des forces d'attraction; réciproquement, un humain sans aucune initiative et intégralement instrumenté par d'autres aura le statut de ressource.

9 Thomas S. Schelling, Micromotives and Macrobehaviour, New York, Norton and Co., 1978. 
etc.

Les types des entités du modèle et leurs relations peuvent tout aussi bien être décrits par une structure mathématique ${ }^{10}$ en termes d'ensembles, de fonctions et d'axiomes; s'il s'agit du diagramme de classe de la figure 1, cette structure est la suivante :

Un ensemble pour chacun des types d'entités, soit :

- un ensemble Individu;

- un ensemble Emplacement, tel que $\mid$ Individu $|<|$ Emplacement $\mid$;

- un ensemble Territoire, tel que $\mid$ Territoire $\mid=1$;

Une fonction pour chacun des services :

- une fonction calculSatisfaction : Individu $\longrightarrow$ \{content, mécontent $\}$;

- une fonction libérer : Individu x Emplacement $\rightarrow$ T;

- etc.

Une relation ou une fonction pour chacune des relations :

- une fonction appartenir : Emplacement $\rightarrow$ Territoire;

- une fonction injective occuper : Individu $\longrightarrow$ Emplacement;

- $\quad$ une relation accessible $\subset$ Individu x Emplacement;

- une relation (symétrique) voisin $\subset$ Emplacement $\mathrm{x}$ Emplacement;

Une fonction pour chacun des attributs des entités :

- une fonction couleur : Individu $\longrightarrow$ \{blanc, noir $\}$;

- etc.

$10 \quad$ Plus précisément une classe d'objets mathématiques. 


\section{Figure 1}

Diagramme de la classe UML du méta-modèle de la ségrégation résidentielle selon Schelling

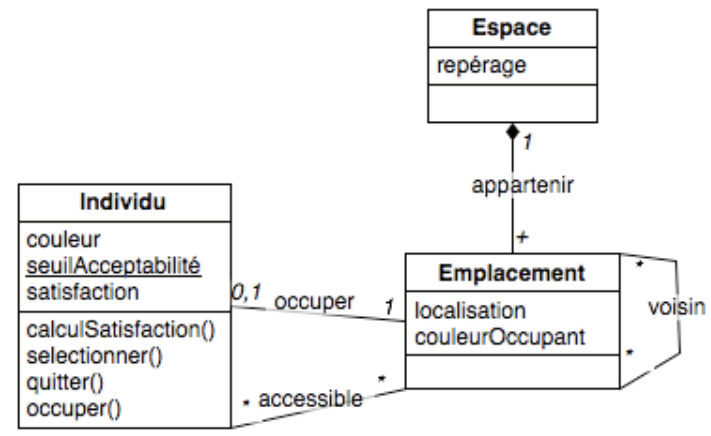

Figure 2

Méta-modèle de la ségrégation résidentielle selon Schelling faisant figurer les transitions

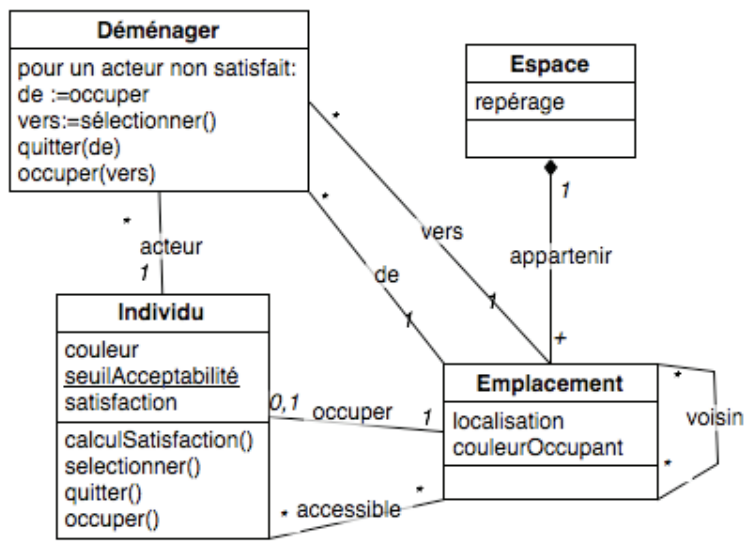

Concernant les transitions, leurs types peuvent soit figurer sur un diagramme de classe tel que celui de la figure 2, soit être décrits séparément.

Ce que nous appellerons la structure du modèle est alors la donnée de :

- l'ensemble des instances des différents types d'entités, ici l'ensemble des Territoires (réduit à un unique élément), 
l'ensemble des Individus et l'ensemble des Emplacements;

- la spécification des services de ces entités, par exemple, sous la forme de pré- et de post-conditions;

- la description de chacun des types de transitions, par exemple, sous la forme d'un algorithme ou d'un ensemble de règles.

Les transitions ne sont pas de même nature que les entités, leurs instances, que l'on appelle plus communément occurrences, étant par nature éphémères, et même instantanées dans le cas des systèmes discrets dont l'état est non observable, ou indéterminé, pendant la durée de l'exécution d'une transition. Cependant, ce sont les entités en présence qui déterminent les types des transitions qui peuvent avoir lieu, et cela justifie de les considérer comme constitutifs de la structure d'un modèle. Ceci dit, considérer que la définition des types de transitions relève de la description du comportement du modèle (voir 2. Comportement d'un modèle) produit, au final, la même description d'ensemble.

Ce que nous appellerons un état du modèle (dont la structure est préalablement définie), c'est la donnée des liens (c'est-à-dire les instances des types de relations) entre les entités ainsi que la valeur de chacun de leurs attributs ${ }^{11}$. Un tel état peut être représenté soit sous la forme d'un diagramme d'objet UML qui soit conforme au diagramme de classe et respecte ses contraintes sémantiques, soit en explicitant, dans la représentation sous forme mathématique, les ensembles et les fonctions correspondant aux relations et aux attributs. L'ensemble des états d'un modèle constitue donc son espace d'états. La séparation entre ce qui relève de la structure d'un modèle et ce qui relève de son état peut paraître arbitraire, en tout état de cause elle met en jeu des choix de modélisation; par exemple, il est bien connu que toute relation peut tout aussi bien être représentée par une entité. Ces

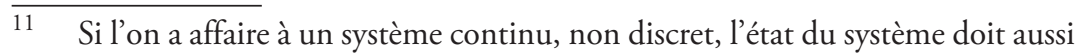
prendre en compte l'état interne des occurrences de transitions qui sont en cours. 
choix de modélisation sont le plus souvent guidés par des critères d'esthétique, dimension essentielle de toute modélisation que nous ne discuterons pas ici, nous contentant de remarquer que ces choix correspondent le plus souvent à des représentations mathématiques qui sont isomorphes.

\section{Comportement d'un modèle}

La définition mathématique de la structure d'un modèle permet de définir des propriétés internes de ce modèle et de les étudier analytiquement. Par exemple, dans le cas du modèle de ségrégation de Schelling, on peut calculer la proportion d'emplacements inoccupés (propriété purement structurelle, indépendante de tout état), ou bien le taux de ségrégation ou la taille des secteurs homogènes pour un état donné.

La réalisation de simulations nécessite que soit définie la dynamique du système, c'est-à-dire comment son état ou sa structure peuvent évoluer.

La structure d'un système permet de définir un ensemble d'actions, à savoir la réalisation par un (ou plusieurs) acteur d'une transition mettant en jeu certaines ressources, dont les occurrences dans un état donné du système sont des actes. Pour simplifier les choses, nous considèrerons ici que les actes modifient l'état du modèle mais ne modifient pas sa structure; ce n'est pas toujours le cas, comme l'illustre, par exemple, la modélisation des configurations sociales de la sociologie de l'imprévisible ${ }^{12}$, mais la prise en compte d'actions qui font évoluer la structure d'un modèle ne change rien à sa présentation, elle est simplement un peu plus compliquée techniquement.

La fonction de transition d'un modèle, qui pour chaque état de son espace d'états indique les (ensembles d') actes qui peuvent se produire et le nouvel état qui résulte de leur réalisation respective, décrit l'ensemble des comportements envisageables du système. Depuis un état initial donné, toute séquence finie

12 Michel Grossetti et Christophe Sibertin-Blanc, « Un méta-modèle des configurations de la sociologie de l'imprévisible ", dans Denis Phan (dir.), Ontologies pour la modélisation multi-agents en sciences humaines et sociales, à paraître en 2010. 
(d'ensembles) d'actes conforme à cette fonction de transition définit une trajectoire dans l'espace d'états du modèle, depuis cet état initial jusqu'à l'état terminal. La définition de cette fonction permet de faire certaines vérifications de la cohérence du modèle, par exemple, par le calcul des états qui sont accessibles depuis un état initial donné. Si le modélisateur a une idée des états initiaux et terminaux qui sont intéressants à considérer, il peut s'assurer que les derniers sont accessibles à partir des premiers. Mais ce n'est généralement pas à partir de cette fonction de transition que sont réalisées des simulations.

En effet, le modèle conceptuel à partir duquel le modèle formel est élaboré comporte une description des processus qui sont à l'œuvre dans le système cible du modèle, ou bien des hypothèses sur ces processus qu'il s'agira de tester. Pour l'essentiel, les résultats de simulation indiquent les effets de ces processus, et ce sont donc eux qu'il s'agit de formaliser pour les mettre en ouvre dans des simulations.

Par définition des trois catégories d'entités qui interviennent dans la structure d'un modèle, ces processus sont portés par les acteurs; chaque acteur du modèle réalise un processus, et le comportement global du système résulte des interactions entre ces processus.

Il s'agit donc, dans un premier temps, de décrire le processus de chacun des acteurs, en fait de chacun des types d'acteurs puisque les acteurs d'un même type suivent, par définition, le même schéma comportemental. Ce processus se déroule toujours selon le cycle :

\section{répéter}

mettre à jour des informations dont l'acteur dispose sur l'état du système

sélectionner un acte (éventuellement de ne rien faire)

réaliser cet acte

jusqu'à la fin

et ce sont donc les étapes de ce cycle qui doivent être décrites, en indiquant comment chaque acteur met à jour ses informations 
et comment il sélectionne le prochain acte qu'il va réaliser ${ }^{13}$.

Il faut ensuite, dans un second temps, décrire comment les processus de chacun des acteurs sont synchronisés les uns avec les autres. Les choses sont relativement simples lorsque chaque acte est réalisé à l'initiative d'un seul acteur. Dans ce cas, s'il n'y a pas une gestion spécifique du temps, soit la simulation fait agir les acteurs de façon asynchrone, comme dans le modèle de ségrégation de Schelling, selon la boucle :

répéter

pour chacun des acteurs sélectionnés

réaliser un cycle

jusqu’à la fin,

soit elle les fait agir de façon synchrone, selon la boucle :

répéter

pour chacun des acteurs sélectionnés

mettre à jour les informations

sélectionner un acte

pour chacun des acteurs sélectionnés

réaliser l'acte sélectionner

jusqu’à la fin.

La synchronisation entre les processus des acteurs est plus délicate à décrire lorsque des actes peuvent être réalisés à l'initiative conjointe de plusieurs acteurs. La synchronisation s'opère alors non plus au niveau des cycles des acteurs comme dans le cas précédent, mais au niveau de l'étape " sélectionner un acte " de ces cycles. Nous n'avons pas étudié cette question, mais il serait certainement intéressant de disposer de mécanismes simples et généraux pour exprimer ce type de synchronisation ${ }^{14}$.

$\overline{13}$ Les acteurs correspondant à des choses (voir note 8), sont généralement déterministes, alors que ceux correspondant à des êtres animés sont le plus souvent dotés d'une certaine autonomie, ils choisissent leur prochain acte parmi un ensemble d'alternatives.

14 On peut remarquer cependant que selon les théories sociologiques de l'action qui mettent en avant les choix rationnels des acteurs sociaux (par exemple, Coleman, Crozier et Friedberg, Boudon, Touraine, Bourdieu pour partie), les interactions entre les acteurs sont médiatisées par des ressources, notamment celles cognitives, dont ils partagent l'accès. Dans ce cas, la décision de 
Il reste enfin à caractériser l'état initial des simulations, y compris la valeur des paramètres qui interviennent dans le comportement de chaque acteur, et le critère de fin des simulations; cela ne présente généralement pas de difficulté. Les simulations se terminent le plus souvent par la satisfaction d'un critère de convergence, l'atteinte d'un état stationnaire ou d'un état particulier, ou encore la durée de la simulation.

\section{Conclusion}

On a exposé dans cet article une certaine façon de présenter des modèles formels destinés à être implantés pour donner lieu à des simulations informatiques, dans le but de faciliter la reproduction des expériences que constituent ces simulations. Cette façon de présenter constitue en fait un formalisme qui est présenté ici de façon informelle, c'est-à-dire sans définition explicite de ses niveaux lexical, syntaxique et sémantique, ce qui serait inutilement technique.

Il convient toutefois de remarquer qu'un modèle formel, même complet et consistant, ne conduit pas automatiquement à des implantations équivalentes, au sens où les mêmes entrées produisent toujours les mêmes sorties. Ce serait compter sans les biais d'implantation qui tiennent aux choix d'implantation du programmeur (inéluctables tant que le code du modèle implanté n'est pas généré automatiquement à partir d'un modèle formel dont la définition est intégralement formalisée), au langage de programmation, aux bibliothèques utilisés, au système d'exploitation de l'ordinateur ou aux caractéristiques physiques de ce dernier. À titre d'exemple, sont en cause la discrétisation d'un phénomène continu, la réalisation de calculs en virgule flottante ou selon l'arithmétique entière, le générateur de nombres aléatoires, ou encore la gestion des fils d'exécutions (threads).

réaliser conjointement une action est elle-même une action, de niveau méta, que chacun réalise pour sa part, et on est ramené au cas où seuls les cycles des acteurs sont alors à synchroniser, les acteurs se coordonnant par l'intermédiaire des ressources. 
Winsberg ${ }^{15}$ étudie certains de ces inévitables biais; Galan et al. ${ }^{16}$ indique comment les mettre en évidence par la réplication, ce qu'elle seule permet de faire, et de préférence en double aveugle. On peut se demander avec Phan ${ }^{17} s^{\prime}$ il est envisageable de produire des modèles formels qui éliminent tous les biais d'implantation, c'est-à-dire dont toutes les implantations correctes soient équivalentes. Nous ne le pensons pas, de même qu'il y a le plus souvent plusieurs façons de formaliser un modèle conceptuel en un modèle formel, il peut y avoir plusieurs façons non équivalentes d'implanter un modèle formel. Le modèle formel est un objet purement symbolique (plus précisément un système de symboles), ce qui n'est le cas ni du modèle implanté ni de son environnement d'exécution. Tout au plus, un modèle formel pourrait-il être accompagné de contraintes d'implantation, faisant alors référence à un état donné de la technologie.

Dans les démarches scientifiques basées sur la simulation, il est fréquent que l'on ne s'intéresse pas à un unique système cible, mais que les résultats soient annoncés comme ayant une portée plus générale et applicables à des systèmes ou phénomènes sociaux différents; il est évident, par exemple, que les résultats associés au modèle de ségrégation de Schelling sont indépendants de la structure concrète du territoire, par exemple, sa taille. Dans ces situations, l'auteur du modèle dispose, implicitement ou non, d'une théorie qu'il applique (ou pourrait appliquer) à divers systèmes concrets pour chacun desquels il est en mesure de produire un modèle conceptuel. Et c'est la parenté entre ces modèles qui fonde la généralisation des résultats à l'ensemble de ces systèmes. Pour cerner cette parenté, et donc la légitimité de cette généralisation, on peut regarder ce qu'il en est des modèles formels de ces différents systèmes. Ce qui justifie la généralisation des résultats, c'est que ces modèles formels partagent le même

15 Eric Winsberg, "Sanctioning Models: The Epistemology of Simulation ", Science in Context, vol. 12, 1999, p. 275-292.

16 José Manuel Galán et al., "Errors and Artefacts in Agent-Based Modelling ", Journal of Artificial Societies and Social Simulation, vol. 12, $\mathrm{n}^{\circ}$ 1, 2009, En ligne : http://www.jasss.soc.surrey.ac.uk/12/1/1.html (le 17 avril 2010).

17 Denis Phan, communication privée. 
méta-modèle - leurs structures étant composées des mêmes types d'acteurs, de ressources et de transitions - et qu'ils ont la même dynamique, les résultats généraux portant sur la nature et l'effet des interactions que peuvent avoir des acteurs des différents types. Pour conclure ce paragraphe, un modèle formel dont on néglige les instances de ses types d'acteurs et de ressources peut être considéré comme une formalisation de la théorie, encore une fois implicite ou non, mise en jeu pour produire le modèle conceptuel dont il est issu, et l'ensemble de ses instanciations comme le domaine d'application de cette théorie.

\section{Bibliographie}

Amblard, Frédéric, Juliette Rouchier et Pierre Bommel, "Évaluation et validation de modèles multi-agents ", dans Amblard, Frédéric et Denis Phan (dir.), Modélisation et simulation multi-agents, applications pour les sciences de l'Homme et de la société, Londres, Hermes-Sciences \& Lavoisier, 2006, p. 103-120.

Axelrod, Robert, "Advancing the Art of Simulation in the Social Sciences ", dans Rosaria Conte, Rainer Hegselmann et Pietro Terna (dir.), Simulating Social Phenomena, Berlin, Springer-Verlag, 1997, p. $21-40$.

Dessalles, Jean-Louis, Jean-Pierre Müller et Denis Phan, "Emergence in Multi-Agent Systems: Conceptual and Methodological Issues ", dans Denis Phan et Frédéric Amblard (dir.), Agent-Based Modelling and Simulation in the Social and Human Sciences, Oxford, The Bardwell Press, 2007, p. 327-356.

Drogoul, Alexi, Diane Vanbergue et Thomas Meurisse, "Multi-Agent Based Simulation: Where Are the Agents? ", dans Jaime Sichman, François Bousquet et Paul Davidsson (dir.), Proceedings of MABS 2002 Multi-Agent-Based Simulation, Lecture Notes in Computer Science 2581, Berlin, Springer-Verlag, 2003, p. 1-15.

Edmonds, Bruce et David Hales, "Replication, Replication and Replication:

Some Hard Lessons from Model Alignment ", Journal of Artificial Societies and Social Simulation, vol. 6, n 4, 2003, En ligne : http://www. jasss.soc.surrey.ac.uk/6/4/11.html (le 17 avril 2010). 
Galán, José Manuel, Luis R. Izquierdo, Segismundo S. Izquierdo, José Ignacio Santos, Ricardo del Olmo, Adolfo López-Paredes et Bruce Edmonds, "Errors and Artefacts in Agent-Based Modelling ", Journal of Artificial Societies and Social Simulation, vol. 12, $\mathrm{n}^{\circ}$ 1, 2009, En ligne : http://www.jasss.soc.surrey.ac.uk/12/1/1.html (le 17 avril 2010).

Grossetti, Michel et Christophe Sibertin-Blanc, "Un méta-modèle des configurations de la sociologie de l'imprévisible ", dans Denis Phan (dir.), Ontologies pour la modélisation multi-agents en sciences humaines et sociales, à paraître en 2010.

Schelling, Thomas S., Micromotives and Macrobehaviour, New York, Norton and Co., 1978.

Vautier, Claude, Pascal Roggero, Françoise Adreit, Christophe SibertinBlanc, "Évaluation by Simulation of the Social Acceptability of Agricultural Policies for Water Quality ", dans Actes de l'International ICSC Symposium on Information Technologies on Environmental Engineering, Thessaloniki (Grèce), mai 2009, Springer Verlag, 2009, p. $478-490$.

Wilensky, Uri et William Rand, « Making Models Match: Replicating an Agent-Based Model ", Journal of Artificial Societies and Social Simulation vol. 10, no 4, 2007, En ligne : http://www.jasss.soc.surrey.ac.uk/10/4/2. html (le 17 avril 2010).

Winsberg, Eric, "Sanctioning Models: The Epistemology of Simulation", Science in Context, vol. 12, 1999, p. 275-292. 